\section{Biomedical Research Awareness Day}

\section{To the Editor:}

My father is a behavioral pharmacologist whose research often involves non-human primates, so as you might imagine, animals in biomedical research was normal and accepted in my family as I was growing up. In fact, it was quite a surprise to me to learn that others were not of the same opinion. How could they not see the benefits of animal research, for humans and for animals? How could they possibly say it isn't worth it? As I began my veterinary career and decided to become a laboratory animal veterinarian, I not only developed a passion for educating others on the importance of biomedical research and the necessity of involving animals in that research, but I felt that it was my obligation to the field and to the animals to tell the truth about animals in research.

However, my impact was limited-I needed an outlet to share the message that so desperately needed to be shared. Shortly after receiving my Doctorate of Veterinary Medicine degree from Texas A\&M University College of Veterinary Medicine \& Biomedical Sciences, I was selected to receive the Michael D. Hayre Fellowship in Public Outreach from Americans for Medical Progress (AMP). With the Fellowship I created Biomedical Research Awareness Day, also known as BRAD, a day to both honor, and raise awareness about, the role of animals in the quest for new treatments and cures for both people and animals.

For BRAD's first year, I focused on veterinary students across the country, urging them to participate by hosting a Biomedical Research Awareness Day celebration at their own institution. Selecting veterinary students as my key audience was intentional, as people often turn to veterinarians for their opinions on a wide variety of issues and tend to take significant stock in their thoughts on animal care, the humane treatment of animals, and the "do's and don'ts" in the animal world. Thus, I thought it was essential that veterinarians, as community educators, understand the importance of biomedical research, humane animal research, and the role of veterinarians in biomedicine and health.

In order to implement BRAD nationwide, each participating school selected a Student Coordinator who communicated with me regularly and spearheaded their BRAD preparations. So as to make it as easy as possible for veterinary students, who are incredibly busy, to participate in BRAD, I provided them with ideas for activities and items to incorporate into their celebration, and I advised them throughout the planning process. One particularly well-received feature of BRAD is its flexibility; each institution was able to tailor their celebration to their specific needs, from a small lunch gathering and discussion to a large celebration involving the public, interactive games, freebies, and educational materials. With the support of AMP, I was able to provide the schools with posters, pens, cups, stickers, interactive games, and other items all geared towards raising awareness about animals in biomedical research.

In its inaugural year, twenty veterinary schools from across the country hosted BRAD celebrations to honor animals involved in biomedical research. Many of the schools created interactive booths where participants could sign a banner pledging their support for animals in research, play the Enrichment Matching Game to pair the proper enrichment item with the animal that uses it, and pick up posters and freebies. Some institutions held seminars covering a variety of topics, from the path to becoming a laboratory animal veterinarian to the canine model of muscular dystrophy and many topics in between. One school even organized a tour of their cat colony, while another incorporated laboratory animal coloring sheets that could then be made into buttons and displayed throughout the day.

Given the success of BRAD's first year, AMP selected three Hayre fellows for the 2016-2017 year, all dedicated to expanding BRAD and making it a sustainable and lasting outreach event. While veterinary schools were the primary audience for the first year, BRAD 2017 is incorporating a much wider audience, including medical and graduate schools, AVMA Accredited, English-speaking international programs, and other institutions that are involved in or support biomedical research. So far, 56 institutions have already signed up for BRAD 2017, more than double the inaugural number last year, and we welcome others!

\section{Logan France}

Johns Hopkins University School of Medicine, Baltimore, MD. Correspondence should be addressed to LFrance5@jhmi.edu. 